\title{
Evidence for Linkage and Association of GABRB3 and GABRA5 to Panic Disorder
}

\author{
Laura M Hodges', Abby J Fyer*,2, Myrna M Weissman ${ }^{2}$, Mark W Logue $^{3}$, Fatemeh Haghighi ${ }^{4}$, Oleg Evgrafov ${ }^{5}$, \\ Allessandro Rotondo ${ }^{6}$, James A Knowles ${ }^{5}$ and Steven P Hamilton*,1,7 \\ 'Department of Psychiatry and Institute for Human Genetics, University of California, San Francisco, CA, USA; ' Department of Psychiatry, \\ Columbia University College of Physicians and Surgeons and the New York State Psychiatric Institute, New York, NY, USA; ${ }^{3}$ Biomedical Genetics, \\ Department of Medicine, Boston University School of Medicine, Boston, MA, USA; ${ }^{4}$ Department of Psychiatry, Icahn School of Medicine, \\ New York, NY, USA; ${ }^{5}$ Department of Psychiatry and Behavioral Sciences, Zilkha Neurogenetic Institute, Keck School of Medicine, University of \\ Southern California, Los Angeles, CA, USA; 'Department of Psychiatry, University of Pisa, Pisa, Italy; 7 Department of Psychiatry, Kaiser \\ Permanente Medical Center, San Francisco, CA, USA
}

\begin{abstract}
Panic disorder (PD) is a debilitating anxiety disorder characterized by episodes of intense fear with autonomic and psychological symptoms that lead to behavioral impairment. A convergence of genetic and biological evidence implicates gamma-aminobutyric acid type A receptor subunits on chromosome $15 q 12$ as candidate genes for PD. This study investigated I20 Caucasian, multiplex PD pedigrees using regional microsatellites (chrl5ql I-13) and found support for linkage (logarithm of odds (LOD) $\geqslant 2$ ), with a prominent parent-of-origin effect. Genotyping with 10 single-nucleotide polymorphisms (SNPs) showed linkage to GABRB3 (rsII63I42I, $L O D=4.6$ ) and GABRA5 (rs20757I6, LOD = 2.2), and allelic association to GABRB3 (rs8024564, $p=0.005 ; r s 8025575, p=0.02$ ) and GABRA5 (rs35399885, $p=0.05$ ). Genotyping of an independent Sardinian PD trio sample also supported association in the region, again with a parent-of-origin effect. These findings provide genetic evidence for the involvement of the genes GABRB3 and GABRA5 in the susceptibility to PD.

Neuropsychopharmacology (2014) 39, 2423-243I; doi:I0.1038/npp.2014.92; published online II June 20I4
\end{abstract}

\section{INTRODUCTION}

Panic disorder (PD) is an anxiety disorder characterized by paroxysmal panic attacks and impairment from the ensuing anticipatory anxiety about recurring attacks. Panic attacks manifest with intense anxiety symptoms such as pounding heart, difficulty breathing, sweating, dizziness, fear of dying, and an urge to flee or escape (Marquez et al, 2001; Starcevic et al, 1993). The lifetime prevalence of PD, including those with and without agoraphobia, is $4.8-5.1 \%$ in the United States (Kessler et al, 2006; Grant et al, 2006), with a 1.9-3.5fold higher prevalence in females, depending upon age (Eaton $e$ t al, 1994). The estimated relative risk to siblings of $\mathrm{PD}$ probands is 5- to 10 -fold higher than the population risk, and the heritablity of PD is 0.48 (Hettema et al, 2001), making PD amenable to gene-mapping efforts. Segregation

\footnotetext{
*Correspondence: Dr AJ Fyer, Department of Psychiatry, Columbia University College of Physicians and Surgeons and the New York State Psychiatric Institute, 1050 Riverside Drive, Unit 82, New York, NY 10032, USA, Tel: +I 212543 5372, Fax: +I 2125436609 , E-mail: ajfyer@pi.cpmc.columbia.edu or Dr SP Hamilton, Department of Psychiatry, Kaiser Permanente Medical Center, 4I4I Geary Boulevard, 3rd Floor, San Francisco, CA 94II8, USA, Tel: +I 4158332034 , Fax: + 415833 2034, E-mail: Steven.P.Hamilton@kp.org Received 30 May 2013; revised 23 February 2014; accepted 24 February 2014; accepted article preview online 23 April 2014
}

studies suggest a complex genetic etiology that reflects phenotypic and locus heterogeneity. In a genome scan of 120 Caucasian multiplex pedigrees, we observed evidence for a susceptibility locus for PD on chromosome $15 \mathrm{q} 12$ (D15S822, two-point heterogeneity logarithm of odds $($ HLOD $)=2.0) \quad($ Fyer et al, 2006). The highest LODtransformed non-parametric linkage score (NPL-LOD), 2.56 , occurred close to the centromere on chromosome 15 and defined a peak of $\sim 12 \mathrm{cM}(1.1-13.6 \mathrm{cM})$ in which NPL-LOD scores were consistently $>2.0$. We carried out empirical significance testing, and the $15 \mathrm{q}$ region achieved an empirical $p$-value of 0.04 for the model-specific analysis. This region is notable for a high density of segmental duplications, the imprinted Prader-Willi/Angelman Syndrome region, and a cluster of GABA receptor subunit genes. In this study, we carried out further mapping of the $15 \mathrm{q}$ region in the same PD sample to narrow the region of linkage by fine-mapping with microsatellites, singlenucleotide polymorphisms (SNPs) at GABRB3 and GA$B R A 5$, followed by mutation screening and parent-of-origin transmission effects in probands by a separate analysis of a Sardinian PD sample. To characterize potential baseline functional variation related to genetic involvement of this region, we also tested genetic effects on familial expression patterns of implicated GABR genes in an unphenotyped sample. 


\section{MATERIALS AND METHODS}

\section{Subject Recruitment and Sample Collection}

A total of 1591 individuals (992 DNA samples) from 120 Caucasian, mulitplex PD pedigrees of primarily Western European descent were recruited from anxiety clinics, therapists, and support groups from the Northeastern United States. Subjects were assessed with the Lifetime Version of the Schedule for Affective Disorders and Schizophrenia (SADS-LA) by blinded clinician interviewers, as described elsewhere (Fyer and Weissman, 1999). A detailed narrative summary for each relative who had panic symptoms was prepared, as was a checklist of medical conditions (including age of onset). At least two senior clinical investigators (AJF, MMW, and Donald F Klein) independently reviewed the case material and diagnosed each individual as to level of affectedness for PD and age of onset. Six levels of PD diagnosis were defined as follows: definite, probable, possible, or any panic, and unaffected, or unknown. These were used to establish three phenotypic designations for statistical analysis such as narrow (definite or probable $\mathrm{PD}$ ), intermediate (adding possible $\mathrm{PD}$ ), and broad (adding any panic) PD phenotypes (Hamilton et al, 1999). All subjects provided informed consent, and the experimental protocol was approved by the institutional review boards of the University of California, San Francisco and the New York State Psychiatric Institute. Genomic DNA was isolated from peripheral blood or lymphoblastoid cell lines as described elsewhere (Knowles et al, 1998).

\section{Sardinian Families}

Trios (PD patients and their biological parents) were recruited from the Department of Psychiatry of the University of Sassari and at the Psychiatric Unit of the General Hospital of Nuoro. Probands were assessed with the SADS-L-Anxiety Version. AR, who had been previously trained in this instrument by our group, supervised translation and back translation of the instrument into Italian and of the case narratives from Italian to English; conducted training sessions with the interviewers in Sardinia to establish diagnostic reliability and teach narrative writing techniques; and carried out ongoing monitoring through review of narratives and site visits to assure reliability and validity of interviews. Case narratives were reviewed for diagnostic consistency by AJF. Cases considered questionable were additionally reviewed by MMW, followed by discussion between the two reviewers to reach a consensus. A total of 49 families (147 individuals) were clinically evaluated with the SADS-L-Anxiety Version and provided DNA samples for this study. Probands were sequentially consenting patients with PD at the two Sardinian clinical sites. The 147 individuals include: 39 parent/child triads $(n=117)$, six proband/parent/sibling triads $(n=18)$, five sibling pairs $(n=10)$, and one proband/ father pair $(n=2)$.

\section{Genotyping by Fragment Analysis and 5' Nuclease Assay}

Microsatellite markers were chosen to cover $\sim 7.14 \mathrm{Mb}$ ( $\sim 19 \mathrm{cM})$ on chromosome 15q11.2-q13.1. We used seven known markers from extant linkage mapping panels, and developed one additional novel polymorphic marker more centromeric than available microsatellites on the long arm of the acrocentric chromosome 15. The average intermarker spacing was $1.02 \mathrm{Mb}$ and $\sim 2.73 \mathrm{cM}$. Genotyping details are described in the Supplementary Methods.

\section{Resequencing Analysis of GABRB3 and GABRA5 in PD Probands}

We performed GABRA5 and GABRB3 mutation screening in a subset of $92 \mathrm{PD}$ probands from the larger set of PD pedigrees, using standard procedures, as described in Supplementary Methods.

\section{SNP Genotyping in Sardinian PD Families}

In the Sardinian PD trios, we used the Illumina GoldenGate assay to type $\sim 335$ evenly spaced SNPs in a $5.34-\mathrm{Mb}$ region within the $15 \mathrm{q}$ region described above. DNA samples from 139 individuals in 49 families, consisting of 34 trios and 15 families of other configurations, were genotyped. Data were output in the form of intensity files and analyzed using Illumina's BEADSTUDIO software. Our final data set included 316 SNPs and 135 samples (97\%) with a mean per sample call rate of $99.7 \%$ and a mean per SNP call rate of $99.8 \%$. Details are provided in the Supplementary Methods. Given the difference in selection processes for each of the genotyping components, there was no overlap of SNP markers between the linkage SNPs and the Sardinian fine-mapping SNPs. We thus genotyped the SNPs from the linkage mapping of US pedigrees in the Sardinian sample.

\section{Linkage and Association Analysis in US PD Pedigrees}

Linkage analysis was performed using several methods corresponding to different underlying biological models for 8 regional microsatellites and 10 evenly spaced SNPs across the genomic region encompassing GABRB3 and GABRA5. First, multipoint LOD-transformed NPL scores were computed, measuring identity-by-descent allele-sharing among affected family members, using GENEHUNTER (Kruglyak et al, 1996). Second, a two-point parametric linkage analysis was performed. Maximum LOD scores for genetic linkage were reported, maximized over mode of inheritance (dominant/recessive) (Greenberg et al, 1998; Hodge et al, 1997), recombination fraction, and the proportion of linked pedigrees under the assumption of locus heterogeneity (HLODs), as is commonly performed in genetic linkage analysis. Parametric assumptions in the models were based upon previous segregation analyses (Vieland et al, 1996), which showed that dominant and recessive single major locus models are equally predictive of the mode of transmission for PD. All LOD scores were computed assuming a $1 \%$ phenocopy rate and $50 \%$ penetrance. Risk allele frequencies under dominant and recessive genetic models were set to and $1 \%$ and $20 \%$, respectively. Based upon sexually dimorphic prevalence rates in PD, unknown or unaffected phenotypes were assigned $66 \%$ and $33 \%$ penetrance, and $14 \%$ and $7 \%$ phenocopy rate in females and males, respectively. Parametric LOD scores were generated using FASTLINK (Cottingham et al, 1993; Schaffer et al, 
1994), and HLODs were computed using HOMOG. A threepoint (genomic position and two flanking markers) parametric linkage analysis incorporating a parent-of-origin effect was computed using LINKAGE-IMPRINT (Shete and Zhou, 2005). For this analysis, the penetrance was set to $50 \%$ and the risk allele frequency was set to $4 \%$. HLODs for the parent-of-origin analysis were computed directly from the by-pedigree LOD scores using R (Ihaka and Gentleman, 1996).

Allelic association analysis in nuclear trios and extended pedigrees was determined using the family-based association test (FBAT) (Laird et al, 2000; Horvath et al, 2001). Multimarker haplotypes were tested for association using FBAT. We report uncorrected $p$-values, owing to the prior evidence for involvement of the $15 \mathrm{q}$ region in $\mathrm{PD}$, although we used permutation to assess empirical significance in selected analyses. Association and parent-of-origin analysis of genotypic data in the Sardinian subset of families was carried out using PLINK (Purcell et al, 2007) and UNPHASED (Dudbridge, 2003).

Parametric linkage, NPL, and allelic association scores were determined for broad, intermediate, and narrow phenotypic definitions for PD. Parent-of-origin linkage analysis was performed using the broad phenotypic definition only.

\section{Genetics of Candidate Gene Expression in CEPH Trios}

We investigated the genetics of GABRA5 and GABRB3 gene RNA expression patterns in cell lines from 30 uncharacterized Caucasian child-parent trios $(n=90)$ of European ancestry from Utah (Coriell Institute for Medical Research, Camden, NJ), for whom genotypic annotation is publicly available via the HapMap Consortium Project (Release 20/ phase II Jan 2006 on NCBI B35 assembly, dbSNP b125, www.hapmap.org). Genotypes for non-redundant SNPs (ie, excluding tagging proxy SNPs at correlation coefficient of $r^{2}=1.0$ ) with minor allele frequencies (MAFs) $\geqslant 0.05$ were tested for association with expression levels using the quantitative transmission disequilibrium test using UNPHASED (Dudbridge, 2003). Details on cell culture conditions and gene expression analysis are described in the Supplementary Methods.

\section{RESULTS}

Our previous work found evidence for linkage between PD and a region of chromosome 15q (Fyer et al, 2006), localized near D15S822, in the proximity of the GABRB3 and GABRA5 genes. We first interrogated the implicated region in chr15q11-13 with eight microsatellites to further refine the linkage interval in the same PD pedigree sample. Several of the eight microsatellites showed suggestive linkage $(\mathrm{LOD} \geqslant 2)$ using two-point analyses under the assumption of heterogeneity. For example, under a recessive model, the two markers, GABRB3 and D15S156, separated by $1.2 \mathrm{Mb}$, showed HLODs $\sim 2$ using broad, intermediate, or narrow definitions of PD (Figure 1). Multipoint analysis largely supported this finding, with a maximal NPL score of 2.93 (NPL-LOD $=1.86, p=0.004)$ under the broad PD diagnostic model at the GABRB3 microsatellite marker. Multipoint parent-of-origin analysis was carried out, and evidence for

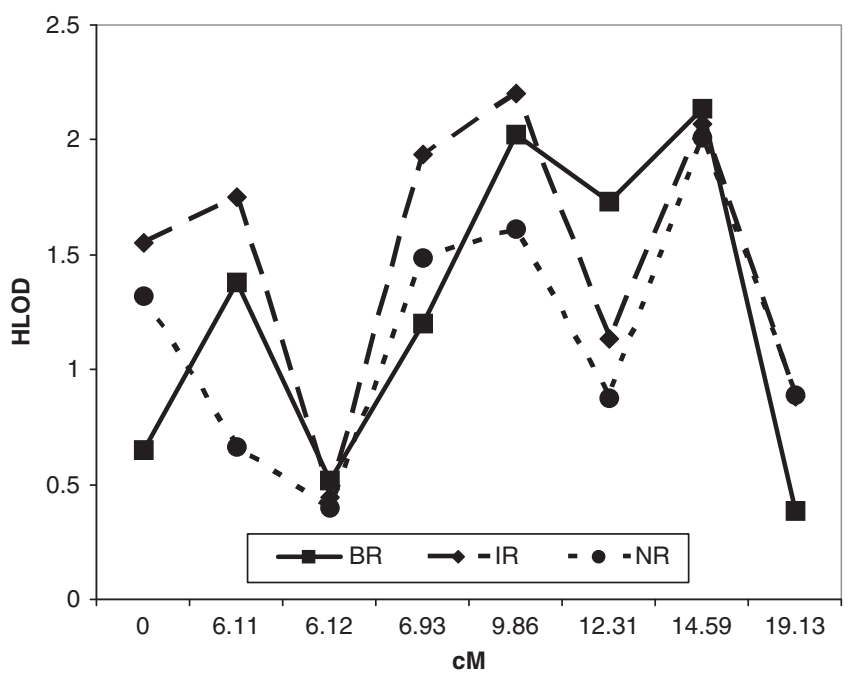

Figure I Fine-mapping of chrl5ql|-13 in 120 multiplex, Caucasian panic disorder (PD) pedigrees by two-point linkage, given locus heterogeneity. $\mathrm{HLOD}=$ heterogeneity logarithm of odds; diagnostic phenotypes are: $\mathrm{B}=$ broad, $\mathrm{I}=$ intermediate, and $\mathrm{N}=$ narrow $\mathrm{PD} ; \mathrm{R}=$ recessive mode of inheritance; $c M=$ centiMorgan.

linkage was found in the region with maximal maternal $\mathrm{HLOD}=3.06$, and a maximum paternal $\mathrm{HLOD}=1.60$, with both scores maximizing at different locations in the interval (Supplementary Figure 1). Given the positional findings, in addition to plausibility as biological candidates, we elected to pursue our findings by additional genotyping using 10 SNPs in the vicinity of the GABRB3 and the neighboring $G A B R A 5$ candidate gene. For two-point parametric analysis, we observed linkage to SNPs in GABRB3 (rs11631421, HLOD $=4.56$ ), as shown in Figure 2 under a recessive model and a broad diagnostic model. For a dominant model, the highest score was found in GABRA5 (rs2075716, HLOD $=2.22$ ), also with a broad diagnostic model (data not shown). Non-parametric multipoint analysis lead to a peak NPL score of 3.70 (NPL-LOD $=2.97, p=0.0008$ ) occurring under a broad diagnostic model on the GABRA5 SNP rs140682, a synonymous SNP occurring in a valine residue conserved among mammals (data not shown).

Nominal allelic association was found for SNPs at GABRB3 (rs8025575, $p=0.005$; rs8024564, $p=0.02$ ) and GABRA5 ( $r 335399885, p=0.05$ ) under a broad diagnostic scheme (Figure 3). Haplotypic analysis did not markedly improve the association signal for the two associated GABRB3 SNPs $(p=0.03)$.

\section{Sardinian Sample}

To further explore association to $\mathrm{PD}$ in the chromosome 15q region, we genotyped 34 trios from Sardinia composed of a proband with PD and biological parents. We genotyped 316 markers across a $5-\mathrm{Mb}$ region centered on the GABA receptor subunit genes. Using the transmission disequilibrium test (Spielman et al, 1993), the marker showing the greatest evidence for association was rs 10220768 $(p=0.003)$, a SNP occurring in a relatively gene-poor region $\sim 400 \mathrm{~kb}$ upstream of the necdin gene (NDN) (Supplementary Figure 2). The next best marker was rs2017247, a SNP lying in the $3^{\prime}$ untranslated region (UTR) of the 

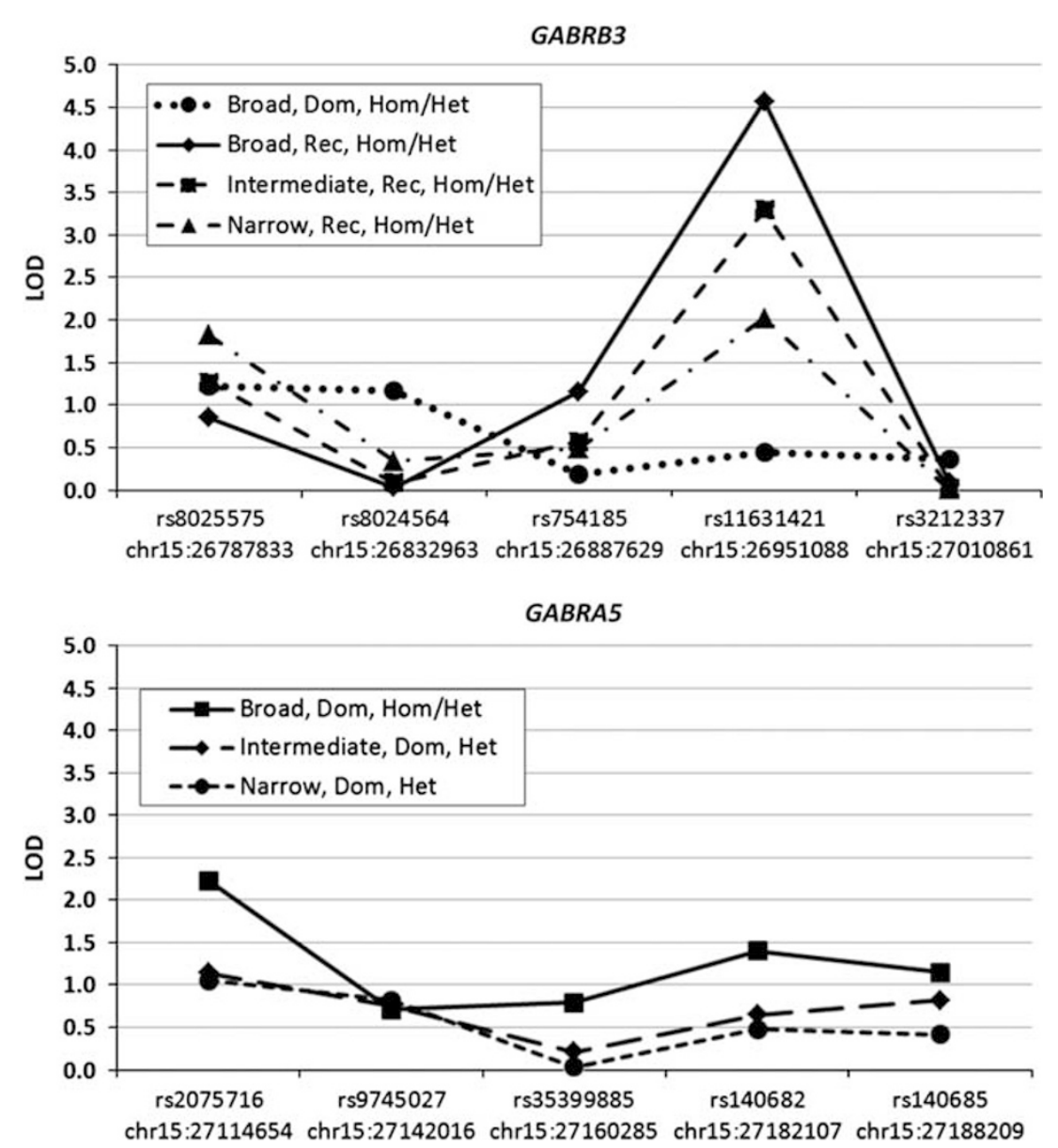

Figure 2 Two-point linkage scores for five single-nucleotide polymorphisms (SNPs) in each of the GABRB3 (upper panel) and GABRA5 (lower panel) genes in 120 multiplex, Caucasian panic disorder (PD) pedigrees from the US logarithm of odds (LOD); SNPs are shown in genomic context as listed in Supplementary Table I. Diagnostic phenotypes are broad, intermediate, and narrow PD; Het=locus heterogeneity; Hom =locus homogeneity; Dom = dominant mode of inheritance; Rec = recessive mode of inheritance; variant position per Feb 2009 (hg 19).

GABRB3 gene $(p=0.004)$. This marker was completely correlated with rs2912586, $5.4 \mathrm{~kb}$ downstream of GABRB3. The permuted pointwise empirical $p$-value for this pair of markers was 0.008 (Table 1). When an additional 15 families were added (mostly discordant sibling pairs with a single biological parent) and the data were analyzed with UNPHASED, the results remained essentially identical. Among the 10 SNPs originally genotyped in our North American PD pedigrees, two GABRB3 markers showed

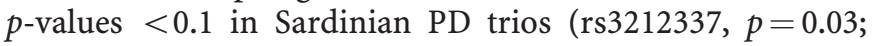
rs11631421, $p=0.07$ ). Given the parent-of-origin effect seen in the linkage analysis from the US pedigrees, and the known complex imprinting status of genes throughout the 15q11-13 region, we considered transmissions from heterozygous parents separately to estimate a parent-of-origin effect in the Sardinian trios. When assessing paternal parentof-origin effect, the best marker was rs2017247 $(p=0.002)$. The pointwise permuted $p$-value was 0.0009 . Of 13 heterozygous fathers, 12 transmitted the risk allele and only one did not (Supplementary Figure 3). Conversely, for 11 heterozygous mothers, the ratio was 7 to $4(p=0.82)$. This marker was also associated with PD in the entire trio set, as described above. The maternal parent-of-origin analysis yielded a best marker with a $p$-value of 0.007 (rs1045935), with a pointwise permuted $p$-value of 0.004 . This SNP occurs in an open reading frame for a primate-specific transcript expressed in brain that is downstream of the SNRPN/SNURF gene complex. The ratio of maternal transmission:non-transmission was 16:4, compared with 9:9 for paternal transmission. The best paternal and maternal findings are almost $1.5 \mathrm{Mb}$ apart.

\section{Mutation Screen in US PD Probands}

A mutation screen of GABRB3 and GABRA5 in a subset of $92 \mathrm{PD}$ probands revealed 9 novel GABRB3 variants (Supplementary Table 4) and 14 novel GABRA5 variants (Supplementary Table 5) of predominantly uncommon $(<0.05)$ MAFs in introns. However, two novel GABRB3 variants in the $3^{\prime}$ UTR of the gene may potentially alter the predicted GATA and NCX (or TLX2 and HOX11L1) transcription factor binding sites (TFBSs). One additional novel GABRB3 indel at chr15:26866266 was detected at high MAF (0.50). In GABRA5, novel variants were observed in the $3^{\prime}$ UTR and $5^{\prime}$ UTR at low MAF. Among the known variants observed in our PD probands, most showed no significant deviation in allele frequency from the publicly available databases for Caucasians except GABRA5 rs41309256 C > T, with MAF of 0.03 in 60 Caucasians according to dbSNP, and 0.13 in PD cases. 
GABRB3
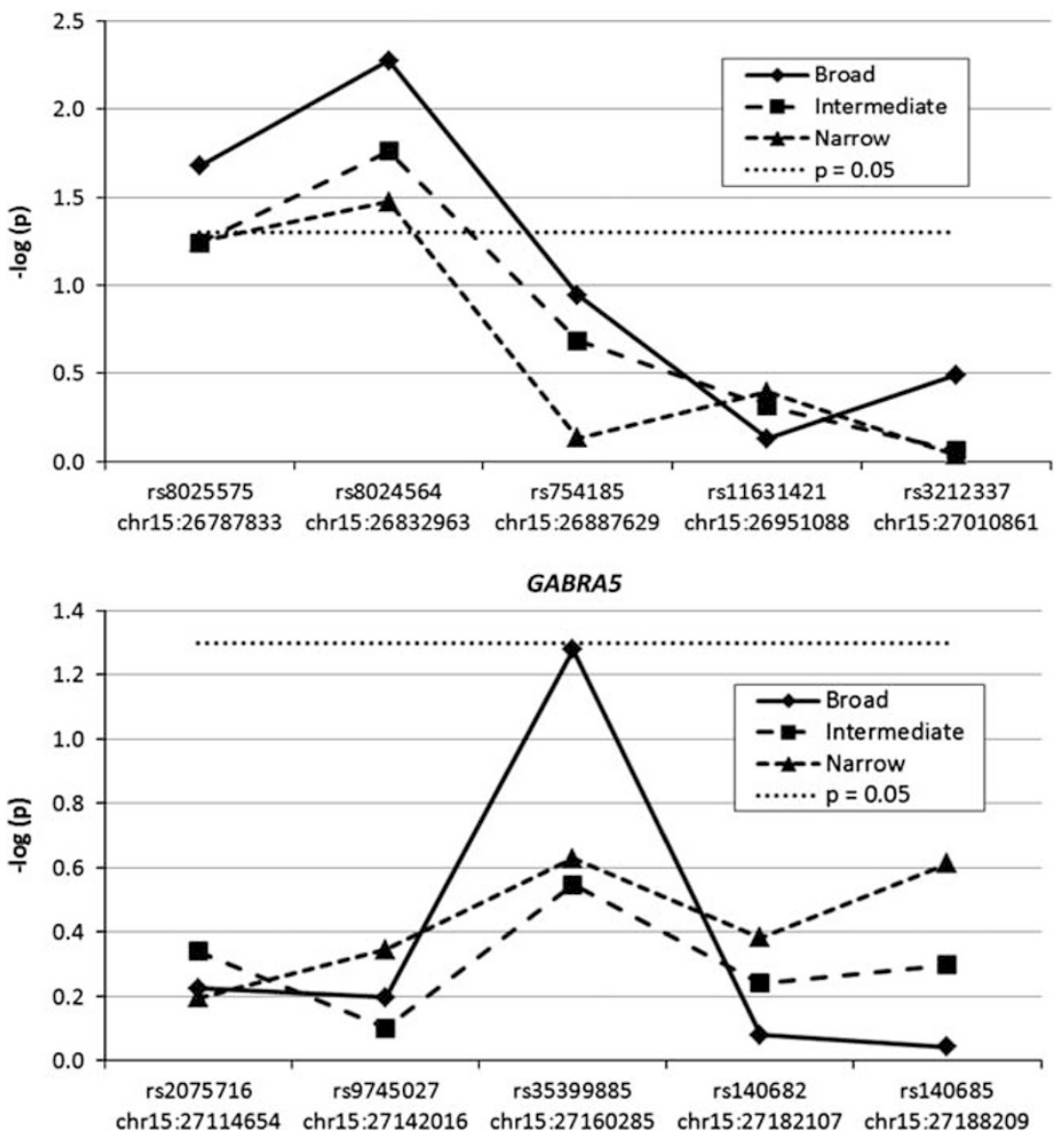

Figure 3 Association analysis for five single-nucleotide polymorphisms (SNPs) in each of the GABRB3 (upper panel) and GABRA5 (lower panel) genes in 120 multiplex, Caucasian panic disorder (PD) pedigrees from the United States. The p-values obtained from family-based association test (FBAT) are depicted by transforming by taking the negative log of the $p$-value. The dotted line depicts a nominal $p$-value of 0.05 . Diagnostic phenotypes are broad, intermediate, and narrow PD; variant position per Feb 2009 (hg 19).

Table I Allelic Association to SNPs in a 5.3-Mb Region Surrounding GABRB3 in 34 Sardinian PD Trios

\begin{tabular}{|c|c|c|c|c|}
\hline SNP & $\begin{array}{c}\text { Position, Feb } 2009 \\
\text { (hgl9) }\end{array}$ & $p($ TDT) & OR $(95 \% \mathrm{Cl})$ & $p($ emp $)$ \\
\hline 10220768 & chr| 5:24319693 & 0.002 & $0.27(0.11,0.67)$ & 0.008 \\
\hline s2912586 & $\operatorname{chr|5:267833||~}$ & 0.004 & $3.80(1.42,10.18)$ & 0.008 \\
\hline rs2017247 & chr|5:26789|62 & 0.004 & $3.80(1.42,10.18)$ & 0.008 \\
\hline rs8029900 & chrl 5:24867594 & 0.007 & $4.00(1.34,11.96)$ & 0.015 \\
\hline rs|29|425| & chr| 5:24889850 & 0.007 & $4.00(1.34,11.96)$ & 0.015 \\
\hline rs|382058 & chrl 5:27758904 & 0.009 & $0.38(0.17,0.8 \mathrm{I})$ & 0.01 \\
\hline rs477847I & chr|5:22964|25 & 0.009 & $3.17(1.27,7.93)$ & 0.018 \\
\hline
\end{tabular}

Abbreviations: OR (95\% Cl), odds ratio of TDT with 95\% confidence interval; $\mathrm{PD}$, panic disorder; $p(E M P)$, pointwise empirical $p$-value after I million permutations; $p(T D T)$, p-value of TDT; SNP, single-nucleotide polymorphism. Position, location at the UCSC genome browser (hg 19, build GRCHr37). Results are shown for SNPs with $p($ TDT) $<0.01$. The SNPs rs29I2586 and rs2017247 show an $r^{2}=1.0$, as rs8029900 and rs 12914251 .

\section{Genetics of GABRB3 Expression in CEPH Trios}

Out of $>23000$ RNA transcripts interrogated on the BeadChip, 6255 were detected in all $90 \mathrm{CEPH}$ cell line samples. GABRA5 expression was below the limit of detection, but GABRB3 was reliably detected in 35 samples derived from 20 unrelated individuals, six parent-child dyads, and one trio. Four of 198 tested SNPs spanning the $G A B R B 3$ locus were associated with significantly altered GABRB3 RNA expression levels (rs2315904, rs10519566, rs7183628, and rs6576613) after 10000 permutations $(p \leqslant 0.01)$ (Table 2). None of these four SNPs were genotyped in our PD samples. The most significantly associated SNP, rs2315904, exhibited a $p$-value of 0.0002 (permutation $p=0.0001$ ) and met strict correction for 198 tests. This SNP lies in an intron of GABRB3.

\section{DISCUSSION}

\section{Chr15q and GABRB3 Variant Association to PD}

In this study, we sought to further characterize the linkage between chromosome $15 \mathrm{q}$ and PD that we observed in our Caucasian sample of 120 multiplex PD pedigrees (Fyer et al, 2006). We found additional support for association between the $15 q$ region and $P D$, despite the very small size of the Sardinian trio sample. When we tested the same 10 SNPs used in the US cohort again in the Sardinian sample, we saw repeated association to GABRB3 SNP rs3212337 in both 
Table 2 Genotypic Association with GABRB3 Expression in Lymphoblastoid Cell Lines from 35 Unphenotyped Caucasians

\begin{tabular}{|c|c|c|c|c|}
\hline SNP & Position, Feb 2009 (hg | 9) & Location & Permuted $p$-value & SNPs captured $r^{2}=1.0 ;$ MAF $=0.05$ \\
\hline rs23I5904 & chrl 5:26967522 & GABRB3 intron & $1.0 \mathrm{E}-04$ & rs23I5903 \\
\hline rs|05|9566 & chrl 5:24526963 & GABRB3 intron & 0.0006 & NA \\
\hline rs7183628 & chrl 5:26995727 & GABRB3 intron & 0.008 & NA \\
\hline rs6576613 & chrl 5:27034566 & $5^{\prime}$ of GABRB3 & 0.006 & rs|2595843, rs749780। \\
\hline
\end{tabular}

Abbreviations: MAF, minor allele frequencies; SNP, single-nucleotide polymorphism.

samples. As in many multistage gene-mapping projects, we have used different SNP markers in different samples, which might have implications for interpretation of results. Our use of different markers for the same targeted region in different samples can be explained by the principles governing genetic analysis in the kind of populations we used, and given the aims we sought to achieve. The use of 8 microsatellites and 10 SNPs targeted to candidate PD genes within the implicated region parsimoniously fulfilled our aim to more precisely pinpoint the linkage signal seen in 120 multiplex, US pedigrees from our previously published work. By their nature, pedigrees contain high linkage disequilibrium in the range with the targeted area that fewer variants were needed capture a signal in this type of sample. However, our aim to corroborate this finding in trios required a greater number of markers as compensation for reduced information and linkage disequilibrium in this type of sample. We genotyped the same 10 fine-mapping SNPs from the US pedigrees in the Sardinian sample that were genotyped for over 300 additional SNPs. In some ways, different markers at the same candidate gene region sufficiently reinforces the hypothesis of the involvement of GABR genes in PD. To illustrate the point further, because so many genetic markers are harbored together in long haplotypes, linkage and association to a given SNP often does not reveal the 'causative' allele. This is a natural limitation of genetic analysis at this scale. Ultimately, future functional studies would be needed to show evidence that a given allele is causal for PD.

\section{Parent-of-Origin Effects}

In both our US and Sardinian samples, we found evidence of parent-of-origin effects. Interestingly, we observed both maternal and paternal parent-of-origin effects that were not overlapping, and consistent with the documented sources of complex pattern of imprinting in this chromosomal region.

\section{GABRB3 and GABRA5 Mutation Screening in PD}

A mutation screen of the GABRB3 and GABRA5 genes found no amino-acid changes but a number of novel synonymous coding variants in both genes in this subset of PD probands. In vitro experiments will be required to determine whether the SNPs discovered in the $3^{\prime}$ and $5^{\prime}$ UTRs of GABRA5 have functional roles, such as translational regulation by altering codon usage or transcriptional regulation by binding sites for enhancers or supressors of expression.

\section{Genetic Association with GABRB3 Expression in CEPH Trios}

To understand possible endophenotypes that may inform putative GABAergic dysregulation in $\mathrm{PD}$, we sought to characterize the expression of the GABA receptor subunit genes in the uncharacterized Caucasian subset of HapMap samples. We performed a test of genetic association with expression levels as a quantitative trait in lymphoblastoid cell lines from CEPH (Centre d'Étude du Polymorphisme Humaine) (Dausset et al, 1990) CEU trios to look for genotypic association with gene expression. We found three intronic SNPs and one SNP lying $5^{\prime}$ to $G A B R B 3$, in the intergenic region between the $5^{\prime}$ ends of GABRB3 and GABRA5, which were associated with GABRB3 expression in lymphoblastoid cell lines. Perfect linkage disequilibrium $\left(r^{2}=1.0\right)$ between the associated rs6576613 variant and untested SNPs (rs12595843 and rs7497801) broaden the implicated region to $\sim 1180 \mathrm{bp}$, which is separated by another $1200 \mathrm{bp}$ from the nearest cluster of predicted TFBSs (Sp-1, NF-YA, and NF-YB). Characterization of genetically mediated GABAergic signaling patterns in PD is needed. Recent magnetic resonance spectroscopy by Long et al (2013) showed significantly lower cortical GABA levels in PD patients than controls, with more exaggerated deficits among patients with a family history of PD.

\section{Continued Support for GABAergic Dysregulation in PD}

The biological role for GABR genes in the pathogenesis of PD is supported by mechanistic, ethological, and clinical evidence. They control neuronal excitability and reduce anxiety via binding to ligands such as benzodiazepines (BZs) used as short-term treatment for PD, as well as to endogenous GABA and neurosteroids. GABRB3 deletion, as in Angelman Syndrome (Holopainen et al, 2001) and knockout mice (Sinkkonen et al, 2003), leads to neurobehavioral effects (DeLorey et al, 1998; DeLorey et al, 2008) and reduced BZ binding. GABRA5 mediates ligand selectivity and affinity (Strakhova et al, 2000) and possibly sedation (van Rijnsoever et al, 2004). PD patients exhibit altered GABA levels (Goddard et al, 2004) and lower BZ binding in the brain (Cameron et al, 2007; Bremner et al, 2000). Panic attacks in PD subjects yield lower than normal levels of neurosteroids (Strohle et al, 2003); whereas PD medications effectively raise central GABA levels (Parent et al, 2002; 
Sanacora et al, 2002; Bhagwagar et al, 2004). Pleiotropic effects of GABAergic dysregulation may explain syndromic features of PD (Weissman et al, 2000; 2004), such as migraine, a common comorbidity also linked to chr15q12 and treated with GABAergic drugs (Russo et al, 2005). Nonequal chromosomal expression (Gimelbrant et al, 2007) or allele-specific expression of disease genes (Tanaka et al, 2012; Pernhorst et al, 2011) such as parent-of-origin expression differences in expression (Kong et al, 2009) can be pathological. For example, maternal overtransmission of the Ser11 allele of GABRB3 rs25409 (C87T, Pro11Ser) results in three- to sixfold greater relative risk for autism (Delahanty et al, 2011). Our data support the hypothesis that genetic variation in the $15 \mathrm{q}$ region, perhaps at GABRB3 and GABRA5, is related to risk for PD and may involve allele-specific expression. However, other studies showed that $G A B R B 3$ and GABRA5 were not associated with PD in a study of 26 pedigrees from the United States and Iceland (Crowe et al, 1997); and linkage analysis (Gelernter et al, 2001; Crowe et al, 2001; Gregersen et al, 2012) and genomewide association studies (Erhardt et al, 2012; Otowa et al, 2012) failed to implicate this region. We view these findings as exploratory, although evidence in more than one $\mathrm{PD}$ sample suggests that GABRB3 and GABRA5 are good candidates to explain at least a portion of the biological and genetic factors in PD pathology.

\section{Parent-of-Origin Findings}

These findings are intriguing, as genes in the chromosome 15q11-13 region are subject to alelle-specific imprinting and large-scale deletions of the non-imprinted maternal or paternal alleles result in Angelman/Prader-Willi syndromes, respectively. The GABRB 3 and GABRA5 genes are largely reported to be biallelically expressed, although there are reports of uniparental expression (Meguro et al, 1997) in cell systems. Parent-of-origin effects have been documented with GABRB3 at the gene expression level in autism (Hogart et al, 2007), as well as at the genotypic level with alcohol dependence (Song et al, 2003). In a subset of our current US PD pedigree collection, we observed nominal parent-of-origin effects for the cumulative lifetime risk for PD when examining patterns of transmission, and noted that the ratio of female to male affected offspring was higher when the disease appeared to be maternally transmitted (Haghighi et al, 1999). No parent-of-origin effects were observed in a smaller number of Italian pedigrees with PD and agoraphobia (Battaglia et al, 1999). However, in our Sardinian trios, we observed parent-oforigin effects that appear to be linked to different areas of chromosome 15, according to either maternal or paternal transmission of PD.

In summary, we found evidence for association and linkage between PD and the region of the GABRB3 and GABRA5 genes in both United States and Sardinian Caucasian families, and a number of novel sequence variants in the region of these two genes among PD probands. In addition, we found support for the genetic contribution to GABRB3 expression in Caucasians, which suggests a possible regulatory mechanism for the hypothesized GABAergic dysregulation in PD. Future studies could further develop these findings with more comprehensive linkage disequilibrium mapping, with additional replication data, and with large-scale sequence analysis across the entire region.

\section{FUNDING AND DISCLOSURE}

This work was supported in part by an award from the Research Evaluation and Allocation Committee of the University of California, San Francisco (to SPH), NIMH Grants MH28274 (to MMW), MH37592 (to AJF), MH30906 (to Donald F Klein), MH48858 (to Susan E Hodge), HG002915 (to FH), MH074118 (to FH), and K01 MH076100 (to MWL). Dr Evgrafov declares that, except for income received from his primary employer and a onetime $\$ 400$ payment from Illumina in 2013 for presentation of data at Illumina workshop, no financial support or compensation has been received from individual or corporate entity over the past 3 years for research or professional service, and there are no personal financial holdings that could be perceived as constituting a potential conflict of interest. Dr Knowles has received research support from the NIH, International OCD Foundation and Della Martin Foundation and speaker's honoraria from Illumina, Inc. Dr Weissman receives royalties from the Oxford University Press, Perseus Press, the American Psychiatric Association Press, and MultiHealth Systems. The remaining authors declare no conflict of interest.

\section{ACKNOWLEDGEMENTS}

We would like to acknowledge the invaluable contribution made by the families who participated in this study. We appreciate the technical assistance of Maria M Bautista, Ramiro Costa, and Rebecca Terrill. We also thank Drs Lorettu (University of Sassari) and Brandano (Psychiatric Unit of the General Hospital of Nuoro) for their recruitment efforts in Sardinia, as well as Dr Donald Klein for participation in the best estimate procedure.

\section{REFERENCES}

Battaglia M, Bertella S, Bajo S, Binaghi F, Ogliari A, Bellodi L (1999). Assessment of parent-of-origin effect in families unlineally affected with panic disorder-agoraphobia. J Psychiatr Res 33: 37-39.

Bhagwagar Z, Wylezinska M, Taylor M, Jezzard P, Matthews PM, Cowen PJ (2004). Increased brain GABA concentrations following acute administration of a selective serotonin reuptake inhibitor. Am J Psychiatry 161: 368-370.

Bremner JD, Innis RB, White T, Fujita M, Silbersweig D, Goddard AW et al (2000). SPECT [I-123]iomazenil measurement of the benzodiazepine receptor in panic disorder. Biol Psychiatry 47: 96-106.

Cameron OG, Huang GC, Nichols T, Koeppe RA, Minoshima S, Rose D et al (2007). Reduced \{gamma\}-aminobutyric acidAbenzodiazepine binding sites in insular cortex of individuals with panic disorder. Arch Gen Psychiatry 64: 793-800.

Cottingham RW Jr., Idury RM, Schaffer AA (1993). Faster sequential genetic linkage computations. Am J Hum Genet 53: 252-263.

Crowe RR, Goedken R, Wilson R, Samuelson S, Nelson J, Noyes JrR (2001). A genome-wide survey of panic disorder. Am J Med Genet (Neuropsychiatr Genet) 105: 105-109. 
Crowe RR, Wang Z, Noyes RJ, Albrecht BE, Darlison MG, Bailey ME et al (1997). Candidate gene study of eight GABAA receptor subunits in panic disorder. Am J Psychiatry 154: 1096-1100.

Dausset J, Cann H, Cohen D, Lathrop M, Lalouel JM, White R (1990). Centre d'etude du polymorphisme humain (CEPH): collaborative genetic mapping of the human genome. Genomics 6: 575-577.

Delahanty RJ, Kang JQ, Brune CW, Kistner EO, Courchesne E, Cox NJ et al (2011). Maternal transmission of a rare GABRB3 signal peptide variant is associated with autism. Mol Psychiatry 16: 86-96.

DeLorey TM, Handforth A, Anagnostaras SG, Homanics GE, Minassian BA, Asatourian A et al (1998). Mice lacking the beta3 subunit of the GABAA receptor have the epilepsy phenotype and many of the behavioral characteristics of Angelman syndrome. J Neurosci 18: 8505-8514.

DeLorey TM, Sahbaie P, Hashemi E, Homanics GE, Clark JD (2008). Gabrb3 gene deficient mice exhibit impaired social and exploratory behaviors, deficits in non-selective attention and hypoplasia of cerebellar vermal lobules: a potential model of autism spectrum disorder. Behav Brain Res 187: 207-220.

Dudbridge F (2003). Pedigree disequilibrium tests for multilocus haplotypes. Genet Epidemiol 25: 115-121.

Eaton WW, Kessler RC, Wittchen HU, Magee WJ (1994). Panic and panic disorder in the United States. Am J Psychiatry 151: 413420.

Erhardt A, Akula N, Schumacher J, Czamara D, Karbalai N, Muller-Myhsok B et al (2012). Replication and meta-analysis of TMEM132D gene variants in panic disorder. Transl Psychiatry 2: e156.

Fyer AJ, Weissman MM (1999). Genetic linkage study of panic: clinical methodology and description of pedigrees. Am J Med Genet 88: 173-181.

Fyer AJ, Hamilton SP, Durner M, Haghighi F, Heiman GA, Costa R et al (2006). A third-pass genome scan in panic disorder: evidence for multiple susceptibility loci. Biol Psychiatry 60: 388-401.

Gelernter J, Bonvicini K, Page G, Woods SW, Goddard AW, Kruger $S$ et al (2001). Linkage genome scan for loci predisposing to panic disorder or agoraphobia. Am J Med Genet 105: 548-557.

Gimelbrant A, Hutchinson JN, Thompson BR, Chess A (2007). Widespread monoallelic expression on human autosomes. Science 318: 1136-1140.

Goddard AW, Mason GF, Appel M, Rothman DL, Gueorguieva R, Behar KL et al (2004). Impaired GABA neuronal response to acute benzodiazepine administration in panic disorder. Am J Psychiatry 161: 2186-2193.

Grant BF, Hasin DS, Stinson FS, Dawson DA, Goldstein RB, Smith $S$ et al (2006). The epidemiology of DSM-IV panic disorder and agoraphobia in the United States: results from the National Epidemiologic Survey on alcohol and related conditions. J Clin Psychiatry 67: 363-374.

Greenberg DA, Abreu P, Hodge SE (1998). The power to detect linkage in complex disease by means of simple LOD-score analyses. Am J Hum Genet 63: 870-879.

Gregersen N, Dahl HA, Buttenschon HN, Nyegaard M, Hedemand A, Als TD et al (2012). A genome-wide study of panic disorder suggests the amiloride-sensitive cation channel las a candidate gene. Eur J Hum Genet 20: 84-90.

Haghighi F, Fyer AJ, Weissman MM, Hodge SE (1999). Parent-oforigin effect in panic disorder. Am J Med Genet B Neuropsychiatr Genet 88: 131-135.

Hamilton SP, Heiman GA, Haghighi F, Mick S, Klein DF, Hodge SE et al (1999). Lack of genetic linkage or association between a functional serotonin transporter polymorphism and panic disorder. Psychiatr Genet 9: 1-6.

Hettema JM, Neale MC, Kendler KS (2001). A review and metaanalysis of the genetic epidemiology of anxiety disorders. $\mathrm{Am} \mathrm{J}$ Psychiatry 158: 1568-1578.
Hodge SE, Abreu PC, Greenberg DA (1997). Magnitude of type I error when single-locus linkage analysis is maximized over models: a simulation study. Am J Hum Genet 60: 217-227.

Hogart A, Nagarajan RP, Patzel KA, Yasui DH, LaSalle JM (2007). 15q11-13 GABAA receptor genes are normally biallelically expressed in brain yet are subject to epigenetic dysregulation in autism-spectrum disorders. Hum Mol Genet 16: 691-703.

Holopainen IE, Metsahonkala EL, Kokkonen H, Parkkola RK, Manner TE, Nagren K et al (2001). Decreased binding of [11C]flumazenil in Angelman syndrome patients with GABA(A) receptor beta3 subunit deletions. Ann Neurol 49: 110-113.

Horvath S, Xu X, Laird NM (2001). The family based association test method: strategies for studying general genotype-phenotype associations. Eur J Hum Genet 9: 301-306.

Ihaka R, Gentleman R (1996). R: a language for data analysis and graphics. J Comput Graph Stat 5: 299-314.

Kessler RC, Chiu WT, Jin R, Ruscio AM, Shear K, Walters EE (2006). The epidemiology of panic attacks, panic disorder, and agoraphobia in the National Comorbidity Survey Replication. Arch Gen Psychiatry 63: 415-424.

Knowles JA, Fyer AJ, Vieland VJ, Weissman MM, Hodge SE, Heiman GA et al (1998). Results of a genome-wide genetic screen for panic disorder. Am J Med Genet B Neuropsychiatr Genet 81: 139-147.

Kong A, Steinthorsdottir V, Masson G, Thorleifsson G, Sulem P, Besenbacher $S$ et al (2009). Parental origin of sequence variants associated with complex diseases. Nature 462: 868-874.

Kruglyak L, Daly MJ, Reeve-Daly MP, Lander ES (1996). Parametric and nonparametric linkage analysis: a unified multipoint approach. Am J Hum Genet 58: 1347-1363.

Laird NM, Horvath S, Xu X (2000). Implementing a unified approach to family-based tests of association. Genet Epidemiol 19(Suppl 1): S36-S42.

Long Z, Medlock C, Dzemidzic M, Shin YW, Goddard AW, Dydak U (2013). Decreased GABA levels in anterior cingulate cortex/ medial prefrontal cortex in panic disorder. Prog Neuropsychopharmacol Biol Psychiatry. 44: 131-135.

Marquez M, Segui J, Garcia L, Canet J, Ortiz M (2001). Is panic disorder with psychosensorial symptoms (depersonalizationderealization) a more severe clinical subtype? J Nerv Ment Dis 189: 332-335.

Meguro M, Mitsuya K, Sui H, Shigenami K, Kugoh H, Nakao M et al (1997). Evidence for uniparental, paternal expression of the human GABAA receptor subunit genes, using microcell-mediated chromosome transfer. Hum Mol Genet 6: 2127-2133.

Otowa T, Kawamura Y, Nishida N, Sugaya N, Koike A, Yoshida E et al (2012). Meta-analysis of genome-wide association studies for panic disorder in the Japanese population. Transl Psychiatry 2: e186.

Parent MB, Master S, Kashlub S, Baker GB (2002). Effects of the antidepressant/antipanic drug phenelzine and its putative metabolite phenylethylidenehydrazine on extracellular gammaaminobutyric acid levels in the striatum. Biochem Pharmacol 63: 57-64.

Pernhorst K, Raabe A, Niehusmann P, van Loo KM, Grote A, Hoffmann $\mathrm{P}$ et al (2011). Promoter variants determine gammaaminobutyric acid homeostasis-related gene transcription in human epileptic hippocampi. J Neuropathol Exp Neurol 70: 1080-1088.

Purcell S, Neale B, Todd-Brown K, Thomas L, Ferreira MA, Bender $\mathrm{D}$ et al (2007). PLINK: a tool set for whole-genome association and population-based linkage analyses. Am J Hum Genet 81: 559-575.

Russo L, Mariotti P, Sangiorgi E, Giordano T, Ricci I, Lupi F et al (2005). A new susceptibility locus for migraine with aura in the 15q11-q13 genomic region containing three GABA-A receptor genes. Am J Hum Genet 76: 327-333. 
Sanacora G, Mason GF, Rothman DL, Krystal JH (2002). Increased occipital cortex GABA concentrations in depressed patients after therapy with selective serotonin reuptake inhibitors. $A m \mathrm{~J}$ Psychiatry 159: 663-665.

Schaffer AA, Gupta SK, Shriram K, Cottingham RW Jr. (1994). Avoiding recomputation in linkage analysis. Hum Hered 44: 225-237.

Shete S, Zhou X (2005). Parametric approach to genomic imprinting analysis with applications to Angelman's syndrome. Hum Hered 59: 26-33.

Sinkkonen ST, Homanics GE, Korpi ER (2003). Mouse models of Angelman syndrome, a neurodevelopmental disorder, display different brain regional GABAA receptor alterations. Neurosci Lett 340: 205-208.

Song J, Koller DL, Foroud T, Carr K, Zhao J, Rice J et al (2003). Association of $\mathrm{GABA}(\mathrm{A})$ receptors and alcohol dependence and the effects of genetic imprinting. Am J Med Genet $B$ Neuropsychiatr Genet 117: 39-45.

Spielman RS, McGinnis RE, Ewens WJ (1993). Transmission test for linkage disequilibrium: the insulin gene region and insulin-dependent diabetes mellitus (IDDM). Am J Hum Genet 52: 506-516.

Starcevic V, Kellner R, Uhlenhuth EH, Pathak D (1993). The phenomenology of panic attacks in panic disorder with and without agoraphobia. Compr Psychiatry 34: 36-41.
Strakhova MI, Harvey SC, Cook CM, Cook JM, Skolnick P (2000). A single amino acid residue on the alpha 5 subunit (Ile215) is essential for ligand selectivity at alpha 5beta 3 gamma 2 gamma aminobutyric acidA receptors. Mol Pharmacol 58: 1434-1440.

Strohle A, Romeo E, di Michele F, Pasini A, Hermann B, Gajewsky $\mathrm{G}$ et al (2003). Induced panic attacks shift \{gamma\}-aminobutyric acid type A receptor modulatory neuroactive steroid composition in patients with panic Disorder: preliminary results. Arch Gen Psychiatry 60: 161-168.

Tanaka M, Bailey JN, Bai D, Ishikawa-Brush Y, Delgado-Escueta AV, Olsen RW (2012). Effects on promoter activity of common SNPs in 5' region of GABRB3 exon 1A. Epilepsia 53: 1450-1456. van Rijnsoever C, Tauber M, Choulli MK, Keist R, Rudolph U, Mohler $\mathrm{H}$ et al (2004). Requirement of \{alpha\}5-GABAA receptors for the development of tolerance to the sedative action of diazepam in mice. J Neurosci 24: 6785-6790.

Vieland VJ, Goodman DW, Chapman T, Fyer AJ (1996). New segregation analysis of panic disorder. Am J Med Genet 67: 147-153. Weissman MM, Fyer AJ, Haghighi F, Heiman GA, Deng Z, Hen $\mathrm{R}$ et al (2000). Potential panic disorder syndrome: clinical and genetic linkage evidence. Am J Med Genet (Neuropsychiatr Genet) 96: 24-35.

Weissman MM, Gross R, Fyer A, Heiman GA, Gameroff MJ, Hodge SE et al (2004). Interstitial cystitis and panic disorder: a potential genetic syndrome. Arch Gen Psychiatry 61: 273-279.

Supplementary Information accompanies the paper on the Neuropsychopharmacology website (http://www.nature.com/npp) 